\title{
Three-Dimensional Analysis of Cracks by Focused Ion Beam and Transmission Kikuchi Diffraction
}

\author{
Majid Abbasi ${ }^{1}$, Ihho Park ${ }^{1}$, Raghavan Ayer ${ }^{1}$, Yunjo Ro ${ }^{1}$ and Hwan-Uk Guim². \\ 1. Global Technology Center, SK Innovation, Daejeon 34124, Republic of Korea. \\ 2. Center for Electron Microscopy Research, Korea Basic Science Institute, Daejeon, Republic of Korea.
}

Investigation of crystallography of crack planes has been a topic of interest for many decades. Understanding how cracks propagate is essential in improving fracture toughness by altering the microstructure and texture. This requires micro-/nano-analysis of grains along the crack path in three dimensions. Electron Backscattered Diffraction (EBSD) on cleavage fracture surfaces or in conjunction with serial-sectioning by Focused Ion Beam (FIB) has been previously utilized to locally study crack crystallography [1-2]. However, the depth of 3D analysis is restricted due to the imposed geometry by FIB serial-sectioning and EBSD $70^{\circ}$ tilt.

Recent developments in Transmission Kikuchi Diffraction (TKD) on electron-transparent samples in SEM offer higher spatial resolution and variable sample tilt [3-7]. Herein, a methodology based on TKD and FIB was explored to investigate crack crystallography by determining crack plane normal and comparing it to crystal orientations. This study was performed on a 2205 duplex stainless steel sample that experienced stress corrosion cracking in service. As presented in Fig. 1a, a FIB slice perpendicular to the crack trace (CT1) is removed. This slice would contain the second crack trace (CT2). The external product of these traces provides the crack plane normal $(\mathrm{CPN}=\mathrm{CT} 1 \times \mathrm{CT} 2)$ as schematically illustrated in Fig. 1b. It is assumed that crack trace (CT1) stays perpendicular to the slice throughout the entire depth.

Maintaining the slice longitudinal direction (A2) perpendicular to the crack path (CT1) and its transverse direction (A1) aligned with the surface normal in Fig. 1a would facilitate the orientation interpretation (see Fig. 1). This way, crystallographic planes could be observed in pole figures with CT1 as normal since the slice normal is parallel to crack path (A3//CT1). An example is shown in Fig. 2. TKD orientation map of the area inside the white box in Fig. 1d is presented in Fig. 2a. Crack traces normal to the slice (CT1) and in the slice (CT2) are marked. Their external product provides CPN as presented with a black arrow in Fig. $2 b$.

The $<111>$ poles of highlighted red austenite and $<011>$ poles of highlighted green ferrite grains along the crack in Fig. 2a are plotted in Fig. 2c and 2d, respectively. The calculated crack plane normal lies within $7^{\circ}$ misorientation of $\langle 111\rangle$ poles in austenite and $10^{\circ}$ misorientation of $\langle 011\rangle$ poles in ferrite (dotted circles in Fig. 2c and 2d). This is an indication of cracking along slip planes in ferrite and austenite. The implications of this observation in the context of stress corrosion cracking mechanism need to be determined. Careful considerations should be taken regarding EBSD/TKD sample coordinate system and FIB slice removal containing cracks [8-12]. A meaningful statistical crack analysis could be achieved by increasing the number of slices. Regarding nanocrystalline samples where many grains are present along cracks, orientation distribution could be utilized instead of discrete pole figures [13].

References:

[1] R. Ayer, R.R. Mueller, T. Neeraj, Mater. Sci. Eng. A 417 (2006), p. 243. 
[2] S. Zaefferer, S.I. Wright, D. Raabe, Metal. Mater. Trans. A 39 (2008), p. 374.

[3] R.H. Geiss, R.R. Keller, D.T. Read, Microsc. Microanal. 16 (2010), p. 1742.

[4] P.W. Trimby, Ultramicroscopy 120 (2012), p. 16.

[5] M. Abbasi et al, ACS Nano 9 (2015), p. 10991.

[6] M. Abbasi et al, Microsc. Microanal. 22 (2016), p. 1652.

[7] J.J. Fundenberger et al, Ultramicroscopy 161 (2016), p. 17.

[8] G. Nolze, Ultramicroscopy 107 (2007) p. 172.

[9] S. Lozano-Perez, Micron 39 (2008), p. 320.

[10] M. Meisnar et al, Micron 75 (2015), p. 1.

[11] T.B. Britton et al, Mater. Char. 117 (2016), p. 113.

[12] T.L. Burnett et al, Scientific Reports 4 (2014), p.4711.

[13] H.G. acknowledges the financial support by the R\&D Convergence Program of National Research Council of Science \& Technology, Republic of Korea (Project Number: CAP-15-02-KBSI).

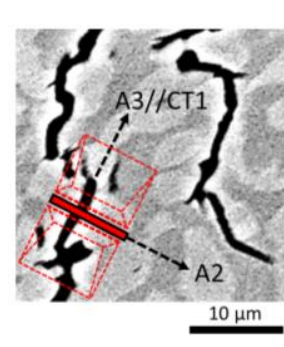

a

Crack before FIB (SE image)

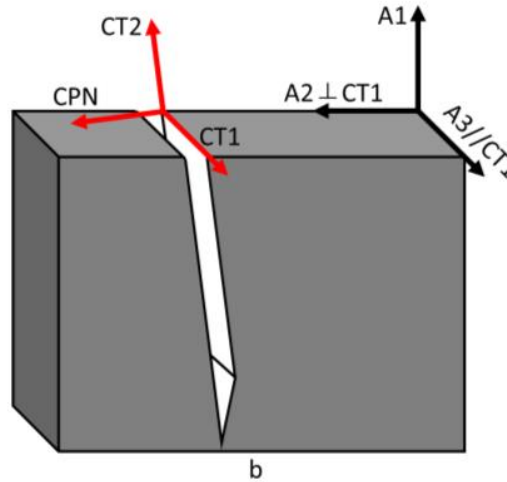

Schematic of FIB slice and crack



c

Actual FIB slice revealing crack path

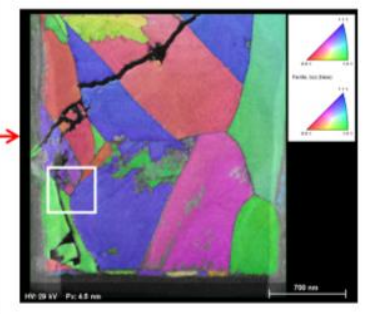

d

Orientation map of grains

Figure 1. a) Secondary electron image of a crack indicating crack trace (CT1) and FIB slice perpendicular to crack path. The top side of FIB slice is depicted by a red bar. Carved trenches are represented as red dashed lines. b) Schematic of the FIB slice, its coordinate system (A1, A2, A3), and crack coordinate system (CT1, CT2, CPN). c) Actual FIB slice revealing the crack path beneath the surface in (a). d) TKD orientation map of austenite and ferrite grains along the crack in FIB slice.



a

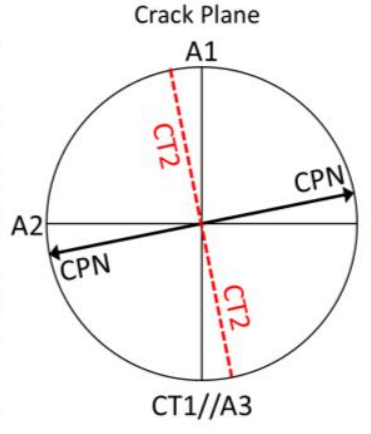

b



c

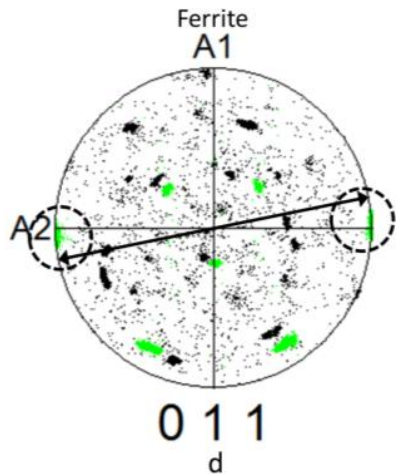

d

Figure 2. a) TKD orientation map of the marked area (white box) in Fig. 1d indicating the subsurface crack and adjacent austenite and ferrite grains highlighted in red and green, respectively. b) Stereographic projection of crack plane normal. c) Crack plane normal with respect to $\langle 111\rangle$ austenite poles in the highlighted red grain. d) Crack plane normal with respect to $\langle 011\rangle$ ferrite poles in the highlighted green grain. CT1 and CT2 as well as CPN are marked in (a). 